\title{
The Design and Characteristic Features \\ of a New Time-of-Flight Mass Spectrometer with a Spiral Ion Trajectory
}

\author{
Takaya Satoh, Hisayuki Tsuno, Mitsuyasu Iwanaga, \\ and Yoshihiro Kammei \\ JEOL Ltd., Tokyo, Japan
}

A new time-of-flight (TOF) mass spectrometer with a corkscrew ion trajectory was designed and constructed. The spiral trajectory was realized by using four toroidal electrostatic sectors. Each had fifteen-stories made of sixteen Matsuda plates piled up inside a cylindrical electrostatic sector. The ions passed the four toroidal electrostatic sectors sequentially and revolved along a figure-eight-shaped orbit on a certain projection plane. During the multiple revolutions, each ion trajectory was shifted by $50 \mathrm{~mm}$ per cycle on a direction perpendicular to the projection plane, thus generating a spiral trajectory. The flight path length of one cycle was $1.308 \mathrm{~m}$ so that the maximum flight path length became $\sim 20 \mathrm{~m}$. The mass resolution, mass accuracy, and ion transmission were tested by utilizing an orthogonally coupled electron ionization source. A mass resolution of 35,000 (FWHM) for $m / z$ greater than 300 was achieved. Even in a lower mass region, mass resolutions of more than 20,000 (FWHM) were confirmed with a doublet of ${ }^{12} \mathrm{C}_{5}^{1} \mathrm{H}_{5}^{14} \mathrm{~N}^{+}$and ${ }^{13} \mathrm{C}^{12} \mathrm{C}_{5}^{1} \mathrm{H}_{6}^{+}$. The mass accuracy was also improved such that it was better than $1 \mathrm{ppm}$ with only one internal standard peak. An ion transmission of approximately of $100 \%$ was observed for 15 cycles. (J Am Soc Mass Spectrom 2005, 16, 1969-1975) (c) 2005 American Society for Mass Spectrometry

A time-of-flight (TOF) mass spectrometer was proposed in the 1940s by Stephens [1] and subsequently developed by Cameron and Eggers [2]. With the advent of matrix-assisted laser desorption/ionization (MALDI) [3, 4] and electrospray ionization (ESI) techniques [5], the TOF mass spectrometer has become a powerful analytical tool, especially in biochemistry and biotechnology. Its characteristic features are high sensitivity, theoretically infinite massrange, and rapid measurements. It also provides a simple and easy method to obtain exact mass measurements. These features give the TOF mass spectrometer a great advantage over other mass spectrometers, such as the quadrupole, ion trap, and magnetic sector-type mass spectrometers. However, a drawback of the TOF mass spectrometer is the intrinsic characteristic of poor mass resolving power for accurate mass analysis, especially for small molecules, compared with a Fourier- transform ion cyclotron resonance (FT-ICR) mass spectrometer. In an orthogonal acceleration (oa-) TOF mass spectrometer, the mass resolution and mass accuracy are limited to approximately 10,000 (FWHM) and 3 ppm, respectively. On the other hand, in the FT-ICR mass spectrometer, they have reached over 100,000 (FWHM) and 1 ppm, respectively.

The mass resolution of the TOF mass spectrometer is

Published online October 24, 2005

Address reprint requests to Dr. T. Satoh, JEOL Ltd., 3-1-2 Musashino, Akishima 196-8558, Tokyo, Japan. E-mail: taksatoh@jeol.co.jp expressed as $R=m / \Delta m=t / 2 \Delta t$, where $t$ is the total time of flight, which is given by the flight path length divided by the ion velocity, and $\Delta t$ is the peak width measured at FWHM. Thus, it is essential to extend the flight path length and minimize the peak width to improve the mass resolution. The peak width depends on the broadening of the ion packet at the detector, especially along the velocity axis and the response time of the detector. Various ion optical techniques have been reported to minimize the peak width: space focusing [6], time-lag focusing [6], orthogonal acceleration [7], and an ion mirror [8] or sector fields [9].

The mass accuracy can be steadily improved by increasing the flight path length under conditions where the bandwidth of the ion detection system is limited. However, the accuracy should be considered for both short and long time periods. The former (0.1-1 $\mathrm{ms})$, corresponds to obtaining one mass spectrum, while the latter ( $1 \mathrm{~h}$ or more), corresponds to a single or sequential LC-MS or GC-MS measurement. Increasing the flight path length is effective in the short time period. To improve the accuracy for the long time period, either internal standards are introduced or the environmental temperature of the mass spectrometer is controlled.

Today, most commercial TOF mass spectrometers are based on linear or reflectron ion optics. Their flight path lengths are several meters and they depend on the size of the instrument. Thus, the method by which the 
flight path length can be increased to a length much greater than the instrument size is crucial for the improvement of mass resolution and mass accuracy.

In 1972, a multi-turn TOF mass spectrometer using electrostatic sector fields was originally proposed by Poschenrieder [9]. In this spectrometer, the flight path length is not restricted to the instrument size and can be infinitely increased because the ions revolve around the same closed orbit. Recently, several new multi-turn TOF mass spectrometers have been constructed in Japan. Sakurai et al. designed and constructed a fairly large multi-turn TOF mass spectrometer referred to as "OVAL" [10] comprising six electrostatic analyzers that produced an elliptical orbit of $7.4 \mathrm{~m}$. Almost simultaneously, a compact multi-turn TOF mass spectrometer referred to as "MULTUM linear plus" [11] was developed at the Osaka University. It consisted of four cylindrical electrostatic sectors and 16 electrostatic quadrupole lenses. The figure-eight-shaped ion orbit had a flight path length of $1.308 \mathrm{~m}$ per cycle. An extremely high mass resolution of 350,000 was reported at $m / z=28$ after ions revolved for 501.5 cycles. "MULTUM II" [12] was developed fairly recently at the Osaka University. In this version, the equipment was simplified by employing only four toroidal electrostatic sectors coupled with four pairs of Matsuda plates [13] and four cylindrical electrostatic sectors. The preliminary results using electron ionization (EI) [12] and MALDI [14] were previously reported.

However, a multi-turn TOF mass spectrometer has a fatal "overtaking" problem because ions having different mass to charge ratios revolve in the closed orbit repeatedly. In due course, the faster ions with smaller $\mathrm{m} / \mathrm{z}$ values pass the slower ones with larger $\mathrm{m} / \mathrm{z}$ values. As a result, ions with different $m / z$ values do not arrive at an ion detector in the order of their $m / z$ value, i.e., the observed mass spectrum deviates from the principle of the TOF mass spectrometer, which states that the detecting time is proportional to the square root of the $\mathrm{m} / \mathrm{z}$ value. Certain complex mathematical treatments are required to transform the deformed mass spectrum into one that is of the order of the $\mathrm{m} / \mathrm{z}$ value.

There are two ways to avoid such complex mathematical treatments. The first, which is an incomplete analysis, requires that only a certain narrow $\mathrm{m} / \mathrm{z}$ range, where no overtaking occurs, should be analyzed. This is because the mass range is inversely proportional to the number of cycles, i.e., the mass range is reduced to $\pm 5 \%$ of the $\mathrm{m} / \mathrm{z}$ value in the case of 10 cycles. The second is the use of a spiral trajectory. The first proposal based on such a spiral trajectory was put forward by Bakker et al. $[15,16]$. Sysoev and coworkers calculated and constructed ion optics using only one electrostatic sector with an ion deflection angle of $509^{\circ}[17,18]$ so that ions travel for approximately 1.5 cycles along a helical ion trajectory. Matsuda also proposed two types of TOF mass spectrometers [19], a corkscrew type and a mosquito-coil type.

To realize a high ion transmission, mass resolution,

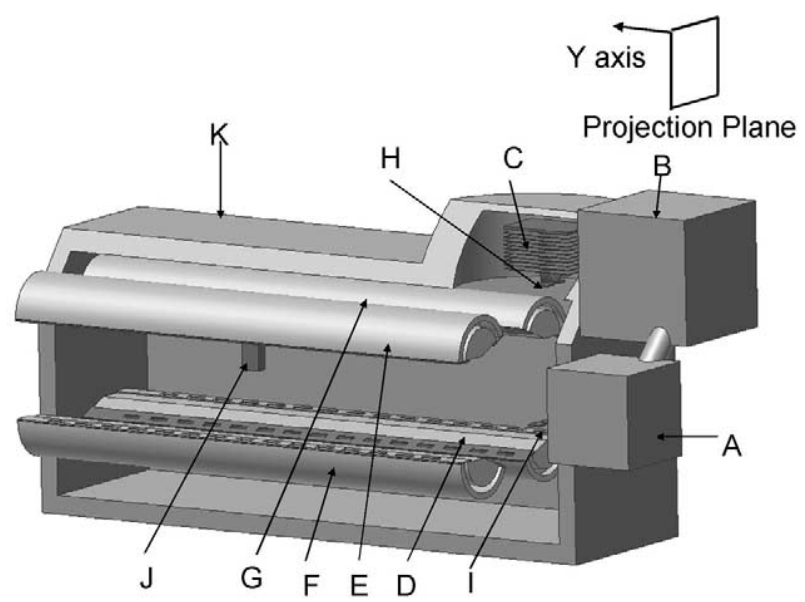

Figure 1. Diagram of the spiral TOF mass spectrometer. (A) Sample reservoir tank; (B) ion source housing; (C) orthogonal acceleration block; (D) toroidal electrostatic sector 1; (E) toroidal electrostatic sector 2; (F) toroidal electrostatic sector 3; (G) toroidal electrostatic sector 4; $(\mathbf{H})$ deflector; (I) beam-defining slit; (J) detector; (K) analyzer housing.

and mass accuracy when increasing the flight path length, it is necessary to design the ion optics to minimize the broadening of the ion packet, not only along the velocity axis but also on the plane perpendicular to it. Matsuda reported the helical ion optics to prevent the broadening of an ion packet on the plane perpendicular to the velocity axis by inserting parallel plate condensers in a field-free region between electrostatic sectors [20]. However, he did not report any details regarding the minimization of the ion packet for the velocity axis in such a case.

We designed and constructed a new spiral TOF mass spectrometer. Its ion optics was designed based on that of MULTUM II with the "perfect focusing" nature [21], which did not cause the broadening of the ion packet at the end of every cycle. The maximum number of cycles was fifteen and the maximum flight path length was approximately $20 \mathrm{~m}$. In this paper, we report the characteristic features of the system and its basic performance, such as mass resolution, mass accuracy, and ion transmission, when an EI source is orthogonally fitted. By increasing the flight path length, a mass resolution of the order of several tens of thousands at $\mathrm{m} / \mathrm{z}<300$ and a mass accuracy better than $1 \mathrm{ppm}$ were expected.

\section{Experimental}

A new spiral TOF mass spectrometer was constructed as an orthogonal acceleration TOF mass spectrometer coupled with an EI source, as schematically shown in Figure 1. The coordinates $(X, Y, Z)$ of the schematics is defined to interpret the acceleration region and ion optics of the mass spectrometer, X-axis shows the direction of a gap of electrostatic sector electrodes, $Y$-axis the shift direction at each cycle that was perpendicular to the projection plane, and Z-axis, the ion 
velocity. The samples were heated in a reservoir tank and introduced into the EI source. The positively charged ions were continuously transferred to an orthogonal acceleration block (oa-block) via an electrostatic lens system. The EI source and electrostatic lens system were arranged at an ion source housing. These positively charged ions were orthogonally accelerated as ion packets toward the spiral ion optics consisting of the four toroidal electrostatic sectors (TESs). The ion packets were injected into the first story of TES 1 at certain a slope angle $\left(\theta_{S L}\right)$ after their ejection angles from oa-block $\left(\theta_{O A}\right)$ were adjusted by a deflector (see Figure 2). Their spatial distributions were limited to the $\mathrm{X}$ - and $\mathrm{Y}$-axis directions by a beam-defining slit. They revolved along the figure-eight-shaped ion orbit on the projection plane while passing TES 1, TES 2, TES 3, and TES 4 sequentially. During the multiple revolutions, each ion trajectory shifted by $50 \mathrm{~mm}$ in the $\mathrm{Y}$ direction per cycle, thus drawing a spiral trajectory. Each TES had fifteen stories and the flight path length of one cycle was $1.308 \mathrm{~m}$, so that the maximum flight path length became approximately $20 \mathrm{~m}$. A movable detector system, by which ions were detected at the center of TES 1 and 4 of every cycle, was mounted. The pressure of the analyzer housing was below $2.0 \times 10^{-5} \mathrm{~Pa}$.

The diagram of the oa-block and deflector are shown in Figure 2. The oa-block comprised a pusher plate, a grounded mesh plate, seven potential divided acceleration plates, and an exit plate. The distance between the pusher plate and grounded mesh plate was $6.5 \mathrm{~mm}$, and that between the grounded mesh plate and exit plate was $52 \mathrm{~mm}$. An ion beam passing through an entrance slit was introduced into the center of the pusher plate and grounded mesh electrode with a kinetic energy of $30 \mathrm{eV}$, and it was orthogonally accelerated with a pulsed voltage applied to the pusher plates. Typically, the entrance slit width was set at $0.7 \mathrm{~mm}$ and the

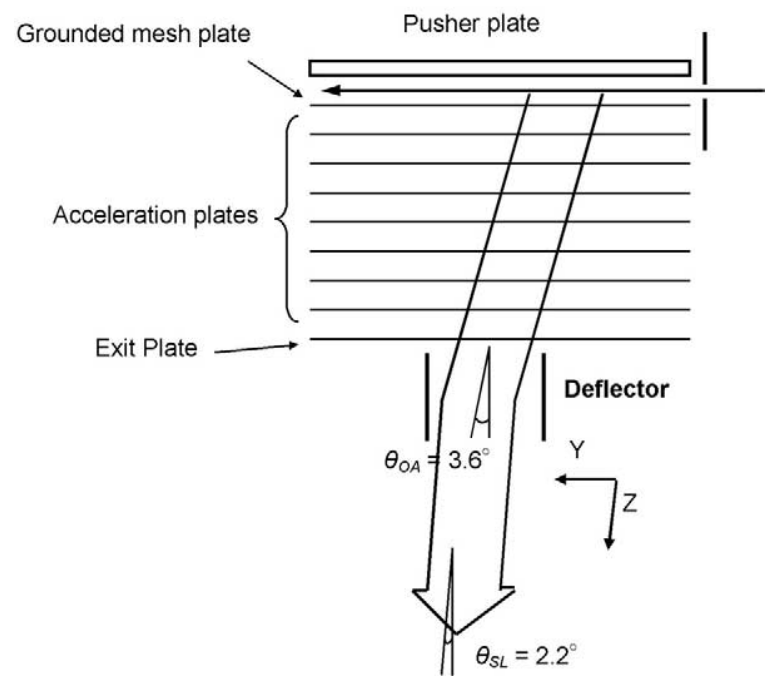

Figure 2. Diagram of the acceleration region of the spiral TOF mass spectrometer. (a)

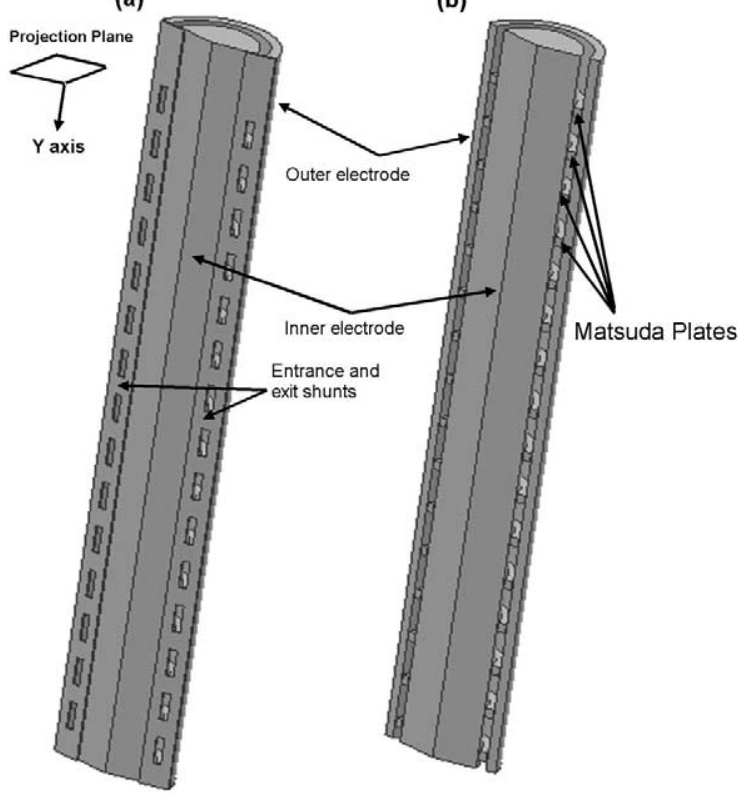

Figure 3. Diagram of the toroidal electrostatic sector 1 (TES 1). (a) With entrance and exit slits and (b) without them. Sixteen Matsuda plates are inserted between the inner and outer electrodes.

amplitude of the pulsed voltage was set at $0.7 \mathrm{kV}$, so that the initial kinetic energy distribution of the ion packet was estimated to be approximately $75 \mathrm{eV}$. After the ions passed the grounded mesh electrode, they were additionally accelerated toward the exit electrode at a potential of $-7.0 \mathrm{kV}$. Thus, the mean ion kinetic energy of the ion packet reached $7.4 \mathrm{keV}$. The ejection angle of the ion packet from the exit electrode $\left(\theta_{O A}\right)$ was approximately $3.6^{\circ}$, which was given by the equation $\theta_{O A}=$ $\tan ^{-1} \sqrt{(30 / 7,400)}$. Then, the angles of the ion packets were adjusted slightly by the deflector to the slope angle of $2.2^{\circ}$ as defined by our design structure of TES, which is described later.

The diagrams of TES 1, with and without entrance and exit shunts, are shown in Figure 3. Each TES comprised inner and outer electrodes, sixteen Matsuda plates, and fifteen entrance and exit shunts. The deflection angle, the radius of the ion trajectory, and the gap between the inner and outer electrodes of the TES were $157.10^{\circ}, 50 \mathrm{~mm}$, and $10 \mathrm{~mm}$, respectively. All the Matsuda plates had a 3D twisted structure. They had a thickness of $10 \mathrm{~mm}$ and were placed with $50 \mathrm{~mm}$ spacing in the $\mathrm{Y}$ direction. The ions travel a distance of $1.308 \mathrm{~m}$ parallel to the orbital surface and shift down by $50 \mathrm{~mm}$ in each cycle, so that the desirable slope angle in the toroidal electrostatic field is $2.2^{\circ}$, from the equation of $\theta_{S L}=\tan ^{-1}(50 / 1308)$. Four TESs were located in point symmetries to form figure-eight-shaped ion orbits on the projection plane. The cross section of the spiral TOF mass spectrometer parallel to the projection plane is shown in Figure 4. The potential of the reference ion trajectory in TES is $-7 \mathrm{kV}$ with respect to the ground 


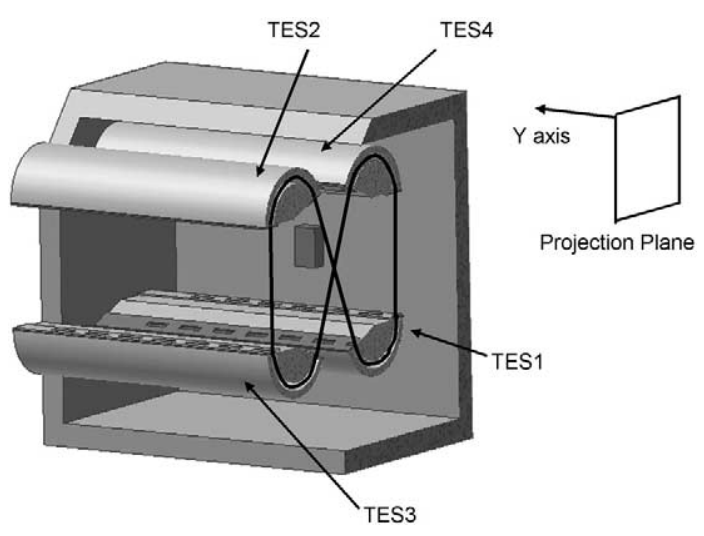

Figure 4. The cross section of spiral TOF mass spectrometer parallel to the projection plane where the ion orbit with a figure-eight-shape can be seen.

potential. The potentials of the inner electrode, outer electrode, and Matsuda plates were $-1.477 \mathrm{kV},+1.477$ $\mathrm{kV}$, and $+0.61 \mathrm{kV}$ from $-7.0 \mathrm{kV}$, respectively.

As mentioned above, the spatial distributions of the ion packets were limited to $1 \mathrm{~mm}$ and $6 \mathrm{~mm}$ in the $X-$ and $\mathrm{Y}$-axis, respectively, by the beam-defining slit located just before the first story of TES 1 . Because it is necessary to strongly maintain the "perfect focusing" nature of MULTUM II on the spiral orbit, the broadening of the ion packets at the detecting surface are assumed to be fairly similar to that of the TES 1 that was first entered. We were concerned about the gain saturation using an $\mathrm{MCP}$, which was currently used as the detector of the TOF mass spectrometer, when the intense ion packets from the EI source were assumed to focus on the limited area of the detector surface. Hence, we used a secondary electron multiplier (P/N 14,882, ETP, Ermington, Australia) as an ion detector in this experiment although its response time of 3 ns was three times longer than that of the MCP.

An 8-bit averager (AP200, Acqiris, Monroe, NY) and a digital oscilloscope (LC584AM, LeCroy, Chestnut Ridge, NY) were used for data acquisition.

\section{Materials}

Perfluorokerosene and a mixture of pyridine and benzene were used. All chemicals were purchased from Kanto Chemical (Tokyo, Japan).

\section{Results and Discussion}

\section{Mass Resolution}

Two spectra of a pyridine and benzene mixture after 15 cycles are shown in Figure 5. Two doublets of ${ }^{12} \mathrm{C}_{5}^{1} \mathrm{H}_{4}^{14} \mathrm{~N}^{+}(m / z=78.0344)$ and ${ }^{12} \mathrm{C}_{6}^{1} \mathrm{H}_{6}^{+}(\mathrm{m} / \mathrm{z}=78.0470)$, and those of ${ }^{12} \mathrm{C}_{5}^{1} \mathrm{H}_{5}^{14} \mathrm{~N}^{+}(\mathrm{m} / \mathrm{z}=79.0422)$ and ${ }^{13} \mathrm{C}^{12} \mathrm{C}_{5}^{1} \mathrm{H}_{6}^{+}$ $(\mathrm{m} / \mathrm{z}=79.0503)$ are shown in Figure $5 \mathrm{a}$ and $\mathrm{b}$, respectively. Since the mass differences of these two doublets are (a) $12.6 \mathrm{mmu}$ and (b) $8.1 \mathrm{mmu}$, the calculated mass resolutions in both cases reach 23,000 (FWHM).
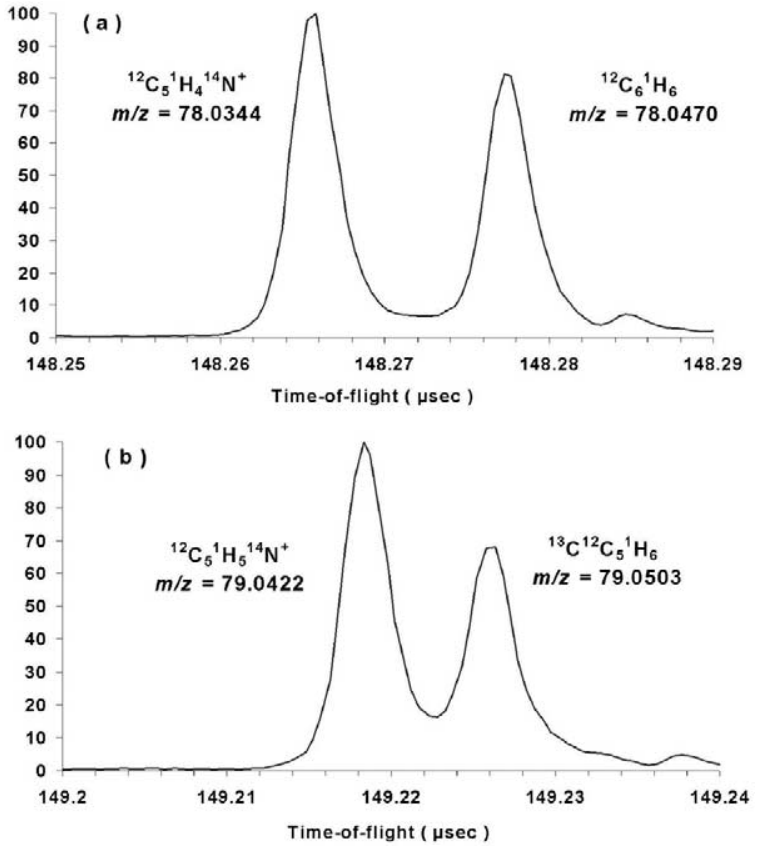

Figure 5. The measured time-of-flight spectra of a mixture of pyridine and benzene at (a) $\mathrm{m} / \mathrm{z}=78$ and (b) $\mathrm{m} / \mathrm{z}=79$.

The relationship between the mass resolution and $\mathrm{m} / \mathrm{z}$ value is shown in Figure 6, where the mass resolutions of several PFK peaks were observed for five, ten, and fifteen cycles. The mass resolutions improve proportionally with the square root of $\mathrm{m} / \mathrm{z}$ value and number of cycles, i.e., the flight path length, when the peak widths are constant. The broadening of the peak width (FWHM) with an increase in the $\mathrm{m} / \mathrm{z}$ values was observed, e.g., the peak widths were slightly broadened from $3 \mathrm{~ns}$ at $\mathrm{m} / \mathrm{z}=69$ to $4.5 \mathrm{~ns}$ at $\mathrm{m} / \mathrm{z}=381$, and the mass resolution at each cycle was improved with an increase in the $m / z$ values. The peak width also became broader to some extent with an increase in the number of cycles. For example, peak widths of $\mathrm{CF}_{3}^{+}(\mathrm{m} / \mathrm{z}=69)$ are $3.1,3.5$, and $3.6 \mathrm{~ns}$ for five, ten, and fifteen cycles, respectively. However, by considering the effect of the extension of flight time, the remarkable ability of preventing the broadening of ion packets must be well presented. In fact, it was observed that the mass reso-

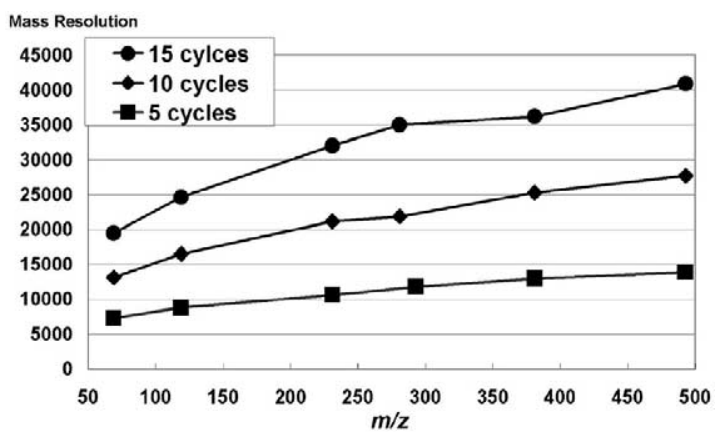

Figure 6. Relationship between the $m / z$ value and mass resolution. 
Table 1. The comparison between the theoretical and calibrated $\mathrm{m} / \mathrm{z}$ value using the different number of terms up to the fifth order for the calibration equations

\begin{tabular}{|c|c|c|c|c|c|c|c|c|c|c|}
\hline \multirow{2}{*}{$\begin{array}{c}\text { Theoretical } \\
m / z\end{array}$} & \multicolumn{2}{|c|}{$1 \mathrm{st}$} & \multicolumn{2}{|c|}{ 2nd } & \multicolumn{2}{|c|}{$3 r d$} & \multicolumn{2}{|c|}{ 4th } & \multicolumn{2}{|c|}{5 th } \\
\hline & $\mathrm{mmu}$ & ppm & $\mathrm{mmu}$ & ppm & $\mathrm{mmu}$ & ppm & $\mathrm{mmu}$ & ppm & $\mathrm{mmu}$ & $\mathrm{ppm}$ \\
\hline 68.9952 & 0.0 & -0.4 & 0.0 & -0.3 & 0.0 & 0.1 & 0.0 & -0.1 & 0.0 & 0.0 \\
\hline 99.9936 & 0.0 & 0.0 & 0.0 & 0.0 & 0.0 & -0.1 & 0.0 & 0.1 & 0.0 & -0.2 \\
\hline 118.9920 & 0.0 & -0.1 & 0.0 & -0.1 & 0.0 & -0.3 & 0.0 & -0.2 & 0.0 & -0.2 \\
\hline 130.9920 & 0.1 & 0.5 & 0.1 & 0.5 & 0.0 & 0.3 & 0.0 & 0.3 & 0.1 & 0.4 \\
\hline 168.9888 & 0.0 & -0.1 & 0.0 & -0.1 & 0.0 & -0.2 & -0.1 & -0.3 & 0.0 & -0.2 \\
\hline 180.9888 & 0.1 & 0.3 & 0.0 & 0.2 & 0.0 & 0.1 & 0.0 & 0.0 & 0.0 & 0.1 \\
\hline 218.9856 & -0.1 & -0.3 & -0.1 & -0.3 & -0.1 & -0.3 & -0.1 & -0.4 & -0.1 & -0.4 \\
\hline 230.9856 & 0.0 & 0.2 & 0.0 & 0.1 & 0.0 & 0.2 & 0.0 & 0.1 & 0.0 & 0.1 \\
\hline 242.9856 & 0.1 & 0.5 & 0.1 & 0.4 & 0.1 & 0.5 & 0.1 & 0.5 & 0.1 & 0.4 \\
\hline 254.9856 & 0.0 & -0.1 & 0.0 & -0.1 & 0.0 & 0.0 & 0.0 & 0.0 & 0.0 & -0.1 \\
\hline 280.9824 & -0.2 & -0.6 & -0.2 & -0.6 & -0.1 & -0.5 & -0.1 & -0.4 & -0.1 & -0.5 \\
\hline 292.9824 & 0.0 & 0.1 & 0.0 & 0.1 & 0.1 & 0.2 & 0.1 & 0.3 & 0.1 & 0.3 \\
\hline 330.9792 & 0.2 & 0.6 & 0.2 & 0.7 & 0.2 & 0.7 & 0.3 & 0.8 & 0.3 & 0.9 \\
\hline 342.9792 & -0.4 & -1.1 & -0.4 & -1.1 & -0.4 & -1.1 & -0.4 & -1.1 & -0.3 & -1.0 \\
\hline 380.9761 & 0.2 & 0.5 & 0.2 & 0.5 & 0.1 & 0.3 & 0.1 & 0.2 & 0.1 & 0.1 \\
\hline $\mathrm{rms}$ & 0.1 & 0.5 & 0.1 & 0.5 & 0.1 & 0.4 & 0.1 & 0.4 & 0.1 & 0.4 \\
\hline
\end{tabular}

lution clearly improves with an increase in the $\mathrm{m} / \mathrm{z}$ value and number of cycles, and that after fifteen cycles, the mass resolution exceeds 35,000 (FWHM) at $m z>$ 300 .

\section{Mass Accuracy}

Exact mass measurements for unknown substances have often been performed with TOF mass spectrometers. Before the measurements, we should determine a calibration equation relating the time-of-flight and the $\mathrm{m} / \mathrm{z}$ value. We attempted to use a polynomial equation with terms up to the fifth order, as shown in eq 1, for the calibration by fitting the measured time-of-flight of the peaks to their theoretical $\mathrm{m} / \mathrm{z}$ values.

$$
\sqrt{m / z}=a+b t+c t^{2}+d t^{3}+e t^{4}+f t^{5}
$$

where $a, b, c, d, e$, and $f$ are constants and $t$ is the time-of-flight. The calibration orders can be selected from the first to the fifth order, i.e., only four constants, $a$ to $d$, need to be determined for the equation with the third-order terms. The comparison between the theoretical and calibrated $\mathrm{m} / \mathrm{z}$ values using the equations with terms from the first- up to the fifth- order is presented in Table 1, wherein the mass deviation among fifteen different masses are also shown in terms of root mean square (rms) values. The mass accuracy is approximately $0.1 \mathrm{mmu}(\mathrm{rms})$ or $0.5 \mathrm{ppm}$ ( $\mathrm{rms}$ ) in all cases. There is no significant difference in the five fitting equations. This implies that the system exhibits a good linearity in the square root of time-of-flight versus the $\mathrm{m} / \mathrm{z}$ value, and that the calibration with four terms up to the third order is sufficient. These results support the fact that the extension of the flight path length is quite effective in improving the mass accuracy.

The dependence of mass accuracy on the number of peaks for calibration was also investigated using five, nine, and fifteen peaks of PFK ions. In the case of using smaller numbers, the other PFK peaks, which are not used for calibration, were regarded as unknown substance peaks. The results are listed in Table 2, where each value was calculated using the fitting equation with four terms up to the third order. All the results are approximately $0.5 \mathrm{ppm}$; this indicates that the mass accuracy was almost independent of the number of peaks for the calibration equation. The fact that only five of the known peaks covering the entire mass range are sufficiently large for exact mass measurements gives us greater freedom to select calibration substances.

In practical measurements, a temporal drift of $\mathrm{m} / \mathrm{z}$ values inevitably occurs along with the thermal expan-

Table 2. The dependence of the mass accuracy on the number of peaks used for the calibration

\begin{tabular}{|c|c|c|c|c|}
\hline \multirow{2}{*}{$\begin{array}{c}\text { Theoretical } \\
m / z\end{array}$} & \multirow{2}{*}{$\begin{array}{l}\text { Used } \\
\text { points }\end{array}$} & $\begin{array}{c}5 \\
\text { peaks }\end{array}$ & \multirow{2}{*}{$\begin{array}{c}9 \\
\text { peaks } \\
\square \text { and } \Delta\end{array}$} & \multirow{2}{*}{$\begin{array}{c}\begin{array}{c}15 \\
\text { peaks }\end{array} \\
\text { All }\end{array}$} \\
\hline & & $\square$ & & \\
\hline 68.9952 & $\square$ & $-0.1 \mathrm{ppm}$ & $0.1 \mathrm{ppm}$ & $0.1 \mathrm{ppm}$ \\
\hline 99.9936 & $\Delta$ & -0.3 & -0.3 & -0.1 \\
\hline 118.9920 & & -0.4 & -0.4 & -0.3 \\
\hline 130.9920 & $\square$ & 0.2 & 0.2 & 0.3 \\
\hline 168.9888 & $\Delta$ & -0.2 & -0.2 & -0.2 \\
\hline 180.9888 & & 0.2 & 0.2 & 0.1 \\
\hline 218.9856 & $\square$ & -0.3 & -0.1 & -0.3 \\
\hline 230.9856 & & 0.2 & 0.4 & 0.2 \\
\hline 242.9856 & & 0.6 & 0.7 & 0.5 \\
\hline 254.9856 & $\Delta$ & 0.1 & 0.3 & 0.0 \\
\hline 280.9824 & & -0.5 & -0.2 & -0.5 \\
\hline 292.9824 & $\square$ & 0.2 & 0.5 & 0.2 \\
\hline 330.9792 & & 0.5 & 0.9 & 0.7 \\
\hline 342.9792 & $\Delta$ & -1.3 & -0.9 & -1.1 \\
\hline \multirow[t]{2}{*}{380.9761} & $\square$ & 0.0 & 0.4 & 0.3 \\
\hline & rms & 0.5 & 0.5 & 0.4 \\
\hline
\end{tabular}


sion of the flight tube and/or fluctuation of electric power supply because of thermal instability. Typically, this drift is not very large, but it is not negligibly small for exact mass measurements. In such a case the internal standard method is preferable. We evaluated the method experimentally by intentionally generating a certain drift value as follows: first, the time-of-flight of PFK ions was measured by applying the voltages of $-1477 \mathrm{~V}$ and $+1477 \mathrm{~V}$ to the inner and outer electrodes, respectively; the constants in the calibration equation with four terms up to the third order were then determined. Second, the two applied voltages were changed to $-1478 \mathrm{~V}$ and $+1478 \mathrm{~V}$ to simulate a temporal drift in the $\mathrm{m} / \mathrm{z}$ values. A comparison between the two is presented in Table 3. All the $\mathrm{m} / \mathrm{z}$ values of the 15 PFK ions were shifted by approximately $630 \mathrm{ppm}$. Finally, assuming the measured peak at the second step (corresponding to $\mathrm{m} / \mathrm{z}=181$ ) to be an internal standard peak, we revised the $\mathrm{m} / \mathrm{z}$ axis by correcting constant $b$ to match the observed and theoretical $\mathrm{m} / \mathrm{z}$ values. The mass deviations of the 15 peaks after calibration are presented in Table 3. The internal calibration was also performed by considering the peak corresponding to $m / z=69$ as an internal calibration peak. This result is also shown in Table 3. In both cases, the mass accuracy was approximately $0.5 \mathrm{ppm}$ (rms), which indicates that exact mass measurements could easily be made for a temporal mass drift correction by using only one internal standard peak.

\section{Ion Transmission}

High sensitivity is one of the most attractive features of a TOF mass spectrometer; hence, the realization of high mass resolution and mass accuracy without appropriate sensitivity is of no significance. The sensitivity of an

Table 3. The mass accuracy using an internal calibration method

\begin{tabular}{|c|c|c|c|}
\hline \multirow{2}{*}{$\begin{array}{c}\text { Theoritical } \\
\mathrm{m} / \mathrm{z}\end{array}$} & \multirow{2}{*}{$\begin{array}{l}\text { Without } \\
\text { correction }\end{array}$} & \multicolumn{2}{|c|}{ Internal calibrant peak } \\
\hline & & $m / z=181$ & $m / z=69$ \\
\hline 68.9952 & $-631.6(\mathrm{ppm})$ & 0.1 (ppm) & $0.0(\mathrm{ppm})$ \\
\hline 99.9936 & -631.3 & 0.3 & 0.2 \\
\hline 118.9920 & -631.7 & -0.2 & -0.3 \\
\hline 130.9920 & -631.4 & 0.1 & 0.0 \\
\hline 168.9888 & -631.8 & -0.3 & -0.4 \\
\hline 180.9888 & -631.4 & 0.0 & -0.1 \\
\hline 218.9856 & -631.5 & -0.1 & -0.2 \\
\hline 230.9856 & -631.5 & -0.2 & -0.3 \\
\hline 242.9856 & -630.8 & 0.6 & 0.5 \\
\hline 254.9856 & -631.7 & -0.3 & -0.4 \\
\hline 280.9824 & -631.6 & -0.2 & -0.3 \\
\hline 292.9824 & -631.7 & -0.4 & -0.5 \\
\hline 330.9792 & -631.1 & 0.2 & 0.1 \\
\hline 342.9792 & -632.4 & -1.1 & -1.2 \\
\hline 380.9761 & -630.1 & 1.2 & 1.1 \\
\hline Average & & -0.01 & -0.12 \\
\hline $\mathrm{rms}$ & & 0.5 & 0.5 \\
\hline
\end{tabular}
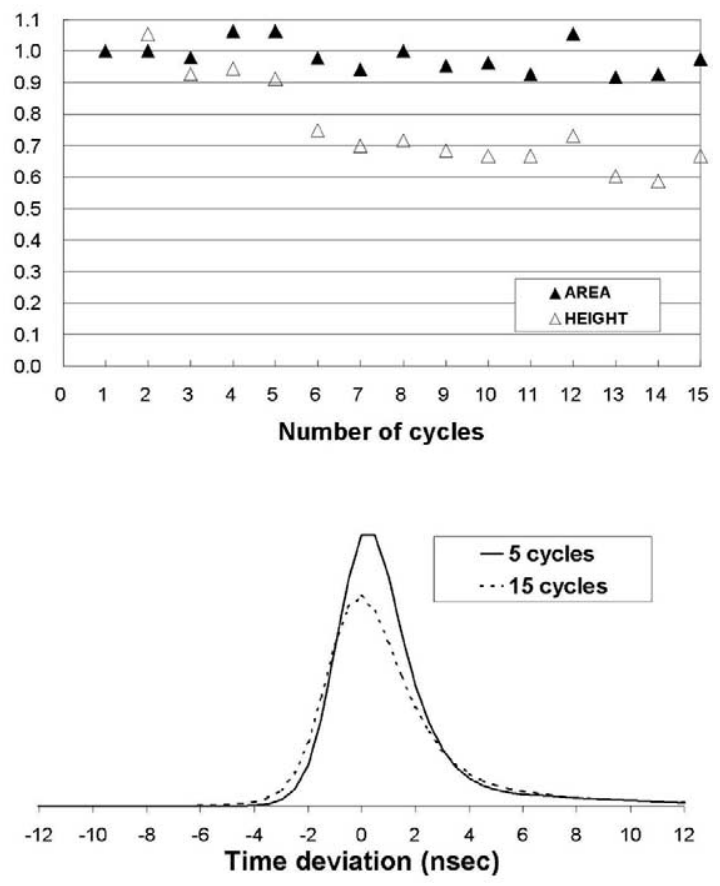

Figure 7. (a) Variation in peak area and height of $\mathrm{CF}_{3}^{+}(\mathrm{m} / \mathrm{z}=69)$ with the number of cycles and (b) the peak shapes in five and fifteen cycles.

oa-TOF mass spectrometer is determined by complicated combinations of ionizing efficiency, ion transmission of low-energy-transfer optics, acceptance and ion transmission of ion optics, detection efficiency of detector, and duty cycle. High ion transmission is extremely important when considering the ion optics.

The ion transmissions and peak shapes in five and fifteen cycles are comparatively shown in Figure 7a and b. The beam-defining slit was fixed at $1 \times 6 \mathrm{~mm}$. The peak heights and areas of $\mathrm{CF}_{3}^{+}(\mathrm{m} / \mathrm{z}=69)$ were measured in one to fifteen cycles. They were normalized by setting each value after one cycle to unity. The areas of the peaks were constant at $100 \%$ with an increase in the number of cycles; in contrast, the peak height reduced to 60 to $70 \%$. These observations indicated a near $100 \%$ ion transmission; however, gradually broadening of ion packet along the velocity axis reduced the peak height to a certain extent. This can be attributed to the accumulation of higher order aberrations of ion optics in accordance with the increasing number of the cycles.

\section{Conclusions}

We designed and constructed a new TOF mass spectrometer with a spiral ion trajectory. In this paper, we reported the major specifications of the system: (1) mass resolution higher than 35,000 for $\mathrm{m} / \mathrm{z}$ values greater than 300 after 15 cycles, (2) mass accuracy better than 1 ppm using the internal calibration method, and (3) nearly $100 \%$ ion transmission.

These results prove that there was no significant increase in the time-focusing aberration compared with 
MULTUM II, and that the perfect focusing property of MULTUM II was maintained for the spiral TOF mass spectrometer as well, if the injection angle toward the TES from the oa-block was selected to be a small value within a few degrees.

\section{Acknowledgments}

The authors are grateful to Professor Emeritus of Osaka University, Hisashi Matsuda, for his encouragement and suggestions through many useful discussions. They also thank Drs. Morio Ishihara and Michisato Toyoda of Osaka University for their support in constructing the system.

\section{References}

1. Stephens, W. E. A Pulsed Mass Spectrometer with Time Dispersion. Phys. Rev. 1946, 69, 691.

2. Cameron, A. E.; Eggers, D. F. An Ion "Velocitron". Rev. Sci. Instrum. 1948, 19, 605-607.

3. Tanaka, K.; Ido, Y.; Akita, S.; Yoshida, Y.; Yoshida, T. Detection of High Mass Molecules by Laser Desorption Time-offlight Mass Spectrometry. Proceedings of the 2nd Japan-China Joint Symposium on Mass Spectrometry; Osaka, Japan, 1987.

4. Karas, M.; Hillenkamp, F. Laser Desorption Ionization of Proteins with Molecular Masses Exceeding 10,000 Daltons. Anal. Chem. 1988, 60, 2229-2301.

5. Yamashita, M.; Fenn, J. B. Electrospray Ion Source; Another Variation on Free-Jet Theme. J. Phys. Chem. 1984, 88, 44514459.

6. Wiley, W. C.; McLaren, I. H. Time-of-Flight Mass Spectrometer with Improved Resolution. Rev. Sci. Instrum. 1955, 26, $1150-1157$.

7. O'Holloran, G. J.; Fluegge R. A.; Betts J. F.; Everett W. J. Report No. ASD-TDR-62-644 Parts 1 and 2: Determination of Chemical Species Prevalent in Plasma Jet. Technical Laboratories Divisions, Southfield Michigan, under Contract Nos. AF33(616)-8374 and AF33(657)-11018. A. F. Materials Laboratory Research and Technology Division, Air Force Systems Command, 1964.

8. Mamyrin, B. A.; Karataev, V. I.; Shmikk, D. V.; Zagulin, V. A. The Mass Reflectron, a New Nonmagnetic Time-of-Flight
Mass Spectrometer with High Resolution. Sov. Phys. JETP 1973, 37, 3745-3748.

9. Poschenrieder, W. P. Multiple-Focusing Time of Flight Mass Spectrometers, Part II. TOFMS with Equal Energy Acceleration. Int. J. Mass Spectrom. Ion Phys. 1972, 6, 357-373.

10. Sakurai, T.; Nakabushi, H.; Hiasa, T.; Okanishi, K. A. New Multi-Passage Time-of-Flight Mass Spectrometer at JAIST. Nucl. Instrum. Methods A 1999, 427, 182-186.

11. Toyoda, M.; Ishihara, M.; Yamaguchi, S.; Ito, H.; Matsuo, T.; Reinhard, R.; Rosenbauer, H. Construction of a New MultiTurn Time-of-Flight Mass Spectrometer. J. Mass Spectrom. 2000, 35, 163-167.

12. Okumura, D.; Toyoda, M.; Ishihara, M.; Katakuse, I. A.Simple Multi-Turn Time of Flight Mass Spectrometer "MULTUM II". J. Mass Spectrom. Soc. Jpn. 2003, 51, 349-353.

13. Matsuda, H. Electrostatic Analyzer with Variable Focal Length. Rev. Sci. Instrum. 1961, 32, 850-852.

14. Okumura, D.; Toyoda, M.; Ishihara, M.; Katakuse, I. Application of a Multi-Turn Time-of-Flight Mass Spectrometer "MULTUM II" to Organic Compounds Ionized by MALDI. J. Mass Spectrom. 2004, 39, 86-90.

15. Bakker, J. M. B. The Time-Focusing Principle. A DoubleFocusing Design for Time-of-Flight Mass Spectrometers. Int. J. Mass Spectrom. Ion Phys. 1971, 6, 291-295.

16. Bakker, J. M. B.; Freer, D. A.; Todd, J. F. J. Preliminary Studies on a New Time-Focusing Time-of-Flight Mass Spectrometer. In Dynamic Mass Spectrometer, Vol. VI; Price, D.; Todd, J. F. J., Eds., Hyden and Son: London, 1981, pp 91-110.

17. Sysoev, A. A. Time-of-Flight Analyzers with Sector Fields: Advances and Prospects. Eur. J. Mass Spectrom. 2000, 6, 501513.

18. Sysoev, A. A.; Sysoev, A. A. Can Laser-Ionization Time-ofFlight Mass Spectrometry be a Promising Alternative to Laser Ablation/Inductively-Coupled Plasma Mass Spectrometry and Glow Discharge Mass Spectrometry for the Elemental Analysis of Solids? Eur. J. Mass Spectrom. 2002, 8, 213-232.

19. Matsuda, H. Spiral Orbit Time of Flight Mass Spectrometer. J. Mass Spectrom. Soc. Jpn. 2000, 48(5), 303-305.

20. Matsuda, H. Improvement of a TOF Mass Spectrometer with Helical Ion Trajectory. J. Mass Spectrom. Soc. Jpn. 2001, 49(6), 227-228.

21. Toyoda, M.; Okumura, D.; Ishihara M.; Katakuse, I. Multi-turn Time-of-Flight Mass Spectrometers with Electrostatic Sectors. J. Mass Spectrom. 2003, 38, 1125-1142. 